\title{
ÉTICAS DE AUTOGOBIERNO BIOMÉDICO: HORIZONTALIDAD Y VERTICALIDAD EN LA ARGENTINA POST-2001
}

\section{Biomedical ethics of self-government: horizontality and verticality in Argentina post-2001}

\author{
Diego Santiago Buttigliero* \\ * Universidad Tecnológica Nacional, Región San Nicolás (Argentina) \\ diegobuttigliero@gmail.com
}

Palabras clave

Crisis social

Salud

Sociedad

Medicina

Psicología social

\section{Keywords}

Social crisis

Health

Society

Medicine

Social psychology

\begin{abstract}
Resumen
Este artículo presenta una experiencia de alteración de las configuraciones de gobierno de un hospital en el marco de la crisis político-social argentina del año 2001. A partir de una metodología de investigación basada en "producciones narrativas" de un grupo de médicos residentes del Hospital Provincial de la República, el texto muestra el trabajo de los agentes de salud en contextos de extrema precariedad y da cuenta de la emergencia de nuevas éticas, prácticas y formas de organización a partir de la conexión con formas de poder que proliferaron desde la base social en el contexto de esa crisis. Estas formas de organización y prácticas permiten la articulación de nuevas éticas como un mecanismo económico clave sobre el cual emerge el marco para pensar nuevas formas de organización y de gobierno del hospital. Se utiliza el concepto de "eticopolítica" con el fin de enfocar la cualidad activa de los sujetos, el grado de reflexión en torno a su acción y la conexión de estos dos factores con la política y las relaciones de poder.
\end{abstract}

\section{Abstract}

This article presents an experience of alteration of governmental configurations in a hospital in the context of the political and social crisis of Argentina in 2001. It does so, based on the analysis of a group of Medical Residents at a Provincial Hospital of the Republic of Argentina through a research methodology of narratives. The text shows the action of health workers in contexts of extreme precariousness and gives an account of the emergence of new ethics, practices and forms of organization through the connection with forms of power that proliferated from the social base in the context of that crisis. Those forms of organization and practice allow the articulation of new ethics as a key economic mechanism over which emerge the frame for new forms of organization and government of the hospital. The concept of "ethopolitics" is used to focus on the active quality of the subjects, the degree of reflection about their action, and the connection of these two factors with politics and power relations.

Buttigliero, D. S. (2017). Éticas de autogobierno biomédico: horizontalidad y verticalidad en la Argentina post-2001. Papeles del CEIC. International Journal on Collective Identity Research, vol. 2017/2, papel 180, CEIC (Centro de Estudios sobre la Identidad Colectiva), UPV/EHU Press, http://dx.doi.org/10.1387/pceic.14217 


\section{INTRODUCCIÓN}

A lo largo de este artículo abordamos una experiencia de alteración de las configuraciones de gobierno dentro del Hospital Provincial de la República Argentina. Más específicamente, analizamos las éticas de autogobierno creadas por un grupo de médicos residentes, las cuales remiten al enfrentamiento de críticas situaciones del sistema de salud luego de las reformas de los 90, así como a la emergencia de nuevas formas de organización y prácticas llevadas adelante por el grupo.

En un primer momento presentamos una introducción histórica a la situación del sistema de salud y especialmente de los hospitales públicos en Argentina, buscando contextualizar la particular experiencia de este grupo de residentes. Destacamos asimismo el papel que ha jugado la ética profesional en la situación posterior a las reformas de los 90 y a la crisis social del año 2001 a partir del concepto de "eticopolítica", señalando a la ética como un elemento clave de una "participación no reglada" (Crojethovic, 2014) de los médicos que permitió salvar del colapso al sistema de salud.

Tras la presentación del enfoque metodológico, bosquejamos el marco institucional del grupo de médicos residentes que hemos investigado entre los años 2003 y 2009. A partir de este punto daremos cuenta de dos formulaciones eticopolíticas diferentes articuladas por los residentes que guían sus prácticas y su experiencia en torno a los términos verticalidad y horizontalidad. Esquematizamos luego tres momentos en los que esas éticas se plasman y se hacen operativas y destacamos su conexión diferencial con una serie de prácticas que caracterizaron las reacciones sociales ante la crisis económica, política y social del año 2001. Focalizamos a continuación el momento de mayor desarrollo de la ética de la horizontalidad y las prácticas sobre las que se sostuvo, señalando especialmente el lugar del grupo de reflexión Balint entre los residentes y sus "confrontaciones" con diversos actores. Por último, señalamos una serie de cualidades que caracterizan a la eticopolítica horizontal de los residentes, a diferencia de la ética verticalista, y que la convierten en un elemento interesante para hacer frente a situaciones de precariedad. 


\section{CRISIS, ÉTICA Y PARTICIPACIÓN INFORMAL}

\subsection{Contexto histórico}

Desde una perspectiva histórica podemos decir que el sistema argentino de salud pública se expandió considerablemente durante los años 40 como consecuencia de las políticas de intervención social del Estado que movilizó el primer gobierno peronista. Esta expansión llevaba como guía los principios de universalidad e integralidad. Sin embargo, a partir de los 50 la crisis fiscal del Estado estimuló una nueva orientación política que redujo la intervención estatal en aquellas áreas susceptibles de ser cubiertas por la actividad privada. En consecuencia, las instalaciones de atención médica del sector público ingresaron a partir de los 60 en un lento proceso de deterioro (Belmartino, 2005). Hacia los años 70 esta evolución implicó prácticamente la resignación de los principios de universalidad y accesibilidad igualitaria para conformar de manera definitiva un modelo basado en la solidaridad grupal gremial en torno a las obras sociales. A ello se sumó la grave situación de crisis que afectó al país a lo largo de los 80 y que desembocó hacia principios de los 90 en una serie de medidas de reforma del Estado que incluían al sector de la salud pero que no representaban una inflexión con respecto al proceso de deterioro.

En lo que refiere a los hospitales públicos, esta reforma significó la implantación del modelo de autogestión hospitalaria a través del cual se buscaba otorgar mayor autonomía y flexibilidad en la gestión de los recursos, en pos de obtener mayores niveles de eficiencia en la gestión interna de dichos hospitales. Dentro de este marco se los autorizaba a complementar los fondos públicos con aquellos originados por contrataciones con empresas privadas de seguros de salud, mutuales y obras sociales, así como a cobrar los servicios de salud a los individuos que tuvieran capacidad de pago. Estos ingresos extrapresupuestarios eran autogestionados directamente por el hospital, para procurar así la descentralización de la administración de los hospitales, complementada por la integración transversal de estos a través de redes de comunicación que coordinaban las unidades.

Esta política de reformas, a pesar de sus buenos augurios, no implicó cambios sustanciales con respecto al proceso de deterioro que se arrastraba desde los años 60. Por un lado, dejó sin resolver las necesidades financieras de los hospitales, con lo cual el sistema de salud 
profundizó aún más su inequidad general. Por otro, persistieron los problemas de escasa eficiencia (ya fuera en materia de egresos hospitalarios como de productividad de los recursos humanos) y de falta de focalización del gasto. En este sentido, algunos autores hablan de una nueva "década perdida" para la salud (Belmartino, 2005).

En este contexto, los hospitales se enfrentaron a un aguzado deterioro generado por la falta de recursos materiales, de personal y de infraestructura edilicia (Cetrángolo y Devoto, 1998; Katz y Muñoz, 1988). Esto promovió un ambiente de incertidumbre y desprotección laboral que determinaba las condiciones de precariedad en las que los trabajadores de la salud llevaban a cabo su labor $y$, por ende, el servicio de salud que brindaban. A su vez se evidenciaba paulatinamente que el aumento del grado de autonomía propuesto hacia adentro de los hospitales no lo era solo respecto a cuestiones y decisiones que se mostraban de poca relevancia, sino que había sido acompañado de una disminución del grado de libertad hacia afuera. Esto es, las decisiones que afectaban a las políticas sanitarias, al manejo del presupuesto nacional, a las compras importantes, o a los nombramientos de cargos -y que hacen al sistema hospitalario en su conjunto- parecían alejarse cada vez más de los trabajadores de la salud (Crojethovic, 2014).

Además, en el año 2000 se desplegó una nueva crisis fiscal que afectó tanto al Estado nacional como a las provincias y resultó en la eclosión, hacia fines de 2001, de una fuerte crisis económico-social. En esos momentos los hospitales fueron afectados por un exacerbado desfinanciamiento estatal, por los efectos del desfasaje ocurrido entre el costo de los insumos en dólares y el presupuesto hospitalario en pesos y por la creciente afluencia de población al hospital, consecuencia del aumento de la desocupación y de la pauperización de las clases medias. Esta situación agudizó aún más la precariedad y el grado de vulnerabilidad institucional que los hospitales venían acarreando, y llegaron a enfrentar la posibilidad de una virtual paralización de la actividad en los hospitales públicos.

\section{2 Ética}

A pesar de la intensa desfinanciación estatal de la salud pública en las reformas de los años 90 que hemos señalado, algunos autores no sitúan ese proceso en el centro del fracaso de dichas reformas (Litewka, 2010). Por el contrario, ponen en el centro los problemas de gobernanza o de 
calidad institucional y focalizan la atención en la escasa transparencia de la administración de los recursos para la salud y más directamente en diversas formas de corrupción. Desde esta posición reivindican las reformas de los 90 sosteniendo que su objetivo central era la justicia distributiva y la promoción de igualdad, lo cual en teoría se operativizaría a partir de una utilización racional y eficaz de los recursos materiales, de mejoras en la gestión y en el manejo de los recursos humanos, de capacitación del personal sanitario y de implantación de criterios de calidad en la producción de los servicios de salud. Plantean entonces que la reforma fue malograda en la práctica por la imposibilidad de modificar el statu quo de las organizaciones y la resistencia de los grupos tradicionales de poder dentro de los modelos médico-hegemónicos. Ello desembocó a su vez en una situación de extrema vulnerabilidad de los hospitales hacia el año 2000.

Testimonios controvertidos ahondan en esta propuesta, como el del sanitarista Ignacio Katz, plasmados en su libro Argentina Hospital (Katz, 2004), en el que narra su experiencia como interventor en un hospital del Gran Buenos Aires a fines de los años 90 dibujando una imagen pesadillesca del hospital público, donde la corrupción reina campante a través de la sobrefacturación, la venta de insumos, el tráfico de sangre y la falta de prestaciones. Asimismo, relata las inmensas dificultades enfrentadas por él en los esfuerzos dirigidos a ordenar la institución y a trasparentar sus actividades.

En este sentido, trabajos como el de Litewka (2010) han señalado que los problemas de gobernanza y corrupción de la institución hospitalaria, aguzados por la ausencia de una bioética capaz de enfrentarlos, impactan en los trabajadores de salud llevándolos a un estado de "atonía ética" (ibídem: 152). En esta atonía la palabra calidad, utilizada reiteradamente en el texto de las reformas, queda vaciada de contenido, mientras que la ética se convierte en un término descontextualizado y relacionado con la frustración y la decepción que conducen al personal médico hacia el desinterés y el cinismo. A este respecto puede destacarse la elevada prevalencia del burnout entre los médicos de Argentina en relación con otros países latinoamericanos (Grau et al., 2009).

La particular frustración de los médicos así como el papel que jugaron la naturaleza y los valores mismos de la profesión en la situación de crisis de la salud pública hacia fines de los 90 han sido reconocidos de diversas 
maneras. La renovación del sentido de la profesión médica se ha señalado como una posible solución a esta situación. Así, por ejemplo, la Revista de Cardiología Argentina público en el año 2010 una "Declaración sobre el profesionalismo médico", retomando una declaración emitida por la Federación Europea de Medicina Interna, el American College of Physicians, la American Society of Internal Medicine y el American Board of Internal Medicine (2010). Al respecto es interesante remarcar la fuerza con que algunos de los principios y las responsabilidades que se proponen en la declaración resuenan con la situación de los hospitales hacia el año 2000.

La ética emerge también en otro sentido y este es el caso del grupo de residentes que analizamos. En este segundo sentido, la ética posibilita la articulación de una acción y de un proyecto grupal. La calificación de ético se sostiene en que se refiere a un conjunto de costumbres y normas que dirigen o valoran el comportamiento humano en una comunidad y, más específicamente, a una construcción grupal, por parte de los residentes, de un conjunto de ideas, percepciones y valoraciones que tácitamente señalan y disputan lo que es bueno, malo, correcto, incorrecto y permitido respecto a una acción. A su vez, este elemento ordena una economía simbólica que desarrolla un plano ético, es decir, donde el bien simbólico imperante, por el que se trabaja y disputa, es ético. Es este un régimen que presupone sujetos libres y reflexivos.

Para abordar esta centralidad de la ética profesional hacemos uso de un concepto que ha sido utilizado para comprender algunas articulaciones de poder en el campo de la salud en las sociedades anglosajonas: el de eticopolítica (Rose, 2001). Este concepto señala la manera en que la ética ha llegado a proveer el "medio" dentro del cual el autogobierno de los individuos puede ser conectado con los imperativos del gobierno bajo el principio liberal de la limitación del poder estatal. Un plano eticopolítico es propio de un modo de gobierno que supone sujetos autónomos sobre cuya acción la acción de gobierno se despliega (Foucault, 1988), y que conforma un gobierno que se legitima a partir de vindicaciones éticas que giran en torno a la concepción de un individuo emprendedor dotado con libertad y autonomía. Un régimen semejante interpela a los médicos y acrecienta especialmente su propia reflexividad y responsabilidad profesional en una dimensión ética individual y grupal (Rose, 2001). En suma, el concepto de eticopolítica permite comprender la cualidad activa de los sujetos, el grado de 
reflexión en torno a su acción y la conexión de estos dos factores con la política y las relaciones de poder.

Desde esta perspectiva podemos caracterizar el caso del grupo de residentes que estudiamos como una eticopolítica resistente que, por un lado, corresponde con el énfasis en la autonomía de los modos de gobierno neoliberales, plasmado en el texto de las reformas de salud de los 90, pero que, por otro lado, y de manera principal, emerge en lo cotidiano del hospital como una práctica de supervivencia que lidia con la situación crítica del sistema de salud y la perspectiva del burnout, manteniendo una relación con los fenómenos sociales emergentes de la crisis del año 2001.

\subsection{Participación informal}

En el caso que hemos estudiado, la ética es un elemento clave que posibilita lo que ha sido llamado "participación no reglada" (Crojethovic, 2014) de los profesionales. Esta es una acción que emerge en los espacios de libertad que median la relación de los sujetos con la estructura organizativa del hospital, donde tiene un papel central la oposición entre la estructura formal de los hospitales públicos y la realidad cotidiana de inestabilidad, precarización y falta de recursos de todo tipo. Esa realidad configura una suerte de "exclusión institucional", por falta de respaldo, de los trabajadores de la salud en el seno del propio sistema de salud pública, de la que forman parte por acción $u$ omisión las autoridades hospitalarias y del ministerio de salud (ibídem).

Es en estos espacios de libertad donde puede llegar a tejerse una solidaridad activa que sustente las iniciativas de participación no reglada. Estas implican el funcionamiento de redes de contactos informales hacia el interior del hospital, y también con otros hospitales, que se extienden como una red de trabajo de "buena voluntad" bajo presupuestos éticos que se ha demostrado imprescindible para sostener la atención y contrarrestar la exclusión del sistema de salud hospitalario. A este respecto, Crojethovic (2014) ha señalado que a través de la participación no reglada los sujetos son empujados por la presión asistencial hacia la toma obligada de decisiones que la constituyen y que esta situación, en el fondo, refleja la obediencia a un poder simbólico que delega la responsabilidad de la asistencia hospitalaria únicamente a manos de los profesionales y trabajadores, desligando al gobierno y a sus autoridades políticas y reproduciendo asimismo la precariedad del 
sistema de salud. Sin embargo, la misma autora también ha afirmado que no todas estas iniciativas no regladas reproducen la precariedad del hospital, sino que algunas, al apuntar a superar el obstáculo inmediato y dotarse de un proyecto, logran introducir cambios no precarios que conducen a resolver la situación de manera más duradera o que incluso logran transformar la reproducción de las estructuras del hospital (ibídem).

Más allá de que la situación de los hospitales a finales de los 90 pueda ser definida por la desfinanciación pública, por problemas de gobernanza o corrupción, por la precariedad laboral, por la libertad y autonomía o aun por el "chantaje ético", la ética profesional aparece como un elemento implicado de manera destacada en estas perspectivas. En el caso que exponemos, la ética demuestra la capacidad para mantener unida una práctica médica frente a situaciones potencialmente disruptivas tales como las que se dieron en la situación que prosiguió a las reformas de los 90 y la crisis de 2001. Una cierta ética profesional se sitúa en el centro del proyecto grupal de los residentes estudiados, que en conjunto constituye un caso de "participación no reglada" que intenta romper con la reproducción de la precariedad hospitalaria. En esta ética destacan las diversas maneras en que los residentes piensan el gobierno del hospital y de sí mismos en tanto profesionales, así como relaciones de poder y políticas particulares, lo cual facilita concebirlas como eticopolíticas distintivas que señalan el enfrentamiento de nuevas situaciones, formas de organización y prácticas llevadas adelante por el grupo.

Pasaremos entonces a exponer las eticopolíticas planteadas por un grupo de médicos residentes del servicio de clínica del Hospital Provincial durante un período que abarca desde el año 2003 al 2009. Antes de ello daremos cuenta de las perspectivas metodológicas que dieron marco a la indagación que fundamenta este artículo.

\section{CONSIDERACIONES METODOLÓGICAS}

El presente texto se basa en una investigación empírica, un estudio de caso (Flyvbjerg, 2004; Van Wynsberghe y Khan, 2007; Yin, 1994) centrado en un grupo de médicos residentes del servicio de clínica del Hospital Provincial de la República Argentina, realizada entre los años 2003 y 2009 desde un enfoque cualitativo. La investigación se centró en el estudio de los significados y las percepciones de las personas 
involucradas, a partir de tareas de tipo observacional, descriptivo, interpretativo y comprensivo. El estudio de caso nos posibilitó enfocar de manera profunda e intensa las tramas de significado y sentido de los médicos residentes tomando las dimensiones sociales como ejes fundamentales en la comprensión del fenómeno y abordando el complejo escenario que configura su contexto. Asimismo, el estudio de caso nos permitió abarcar tanto lo singular de la situación del grupo como su complejidad, sin perder de vista las conexiones y la posibilidad de transferir lo aprendido hacia otros casos (Guba y Lincoln, 1994).

Para desarrollar esta indagación nos hemos apoyado, por un lado, en la técnica de las "producciones narrativas" (Balasch y Montenegro, 2003; Pujol, Montenegro y Balasch, 2003) y, por otro lado, en la propia experiencia del autor en la coordinación psicológica del espacio de reflexión de este grupo de residentes, el llamado Grupo Balint (Ribé Buitrón, 2012). La coordinación se realizó durante los años 2007, 2008 y 2009 y fue plasmada sistemáticamente en el cuaderno de notas'.

Las "producciones narrativas", fundamentadas en la perspectiva de los "conocimientos situados" (Haraway, 1995), permitieron generar un conjunto de textos a partir de una conversación igualitaria y localizada, y un trabajo compartido entre el investigador y los participantes, que expresaban sus posicionamientos. En el proceso que propone esta técnica, el análisis comienza de manera conjunta en la producción de las narrativas de los participantes y continúa con la narración del investigador, que es circularmente remitida a los participantes para obtener su aval o críticas y ser posteriormente enriquecida. No se trata de representar al participante y su opinión o a un fenómeno dado, sino de articularse con los participantes para generar un conjunto de textos que configuran nuevos espacios de comprensión y producción de significados. Estos significados remiten a una objetividad abierta y parcial, desde una posición no esencializada y responsable de los efectos de poder que se desprenden de la producción del conocimiento. A su vez, la elección de las producciones narrativas como metodología se debe a las resonancias que esta presenta con los aspectos eticopolíticos que singularizan al grupo de residentes.

\footnotetext{
${ }^{1}$ Agradezco a la psicóloga Cecilia Mosquera, quien asumió el rol de observadora participante de las coordinaciones del grupo de reflexión de residentes y que metódicamente plasmó las interacciones grupales en el cuaderno de notas.
} 
De tal forma, elegimos para realizar en conjunto las narrativas a tres médicos residentes que participaron de diferentes momentos del grupo durante el período consignado²: Mariana, que ingresó en el año 2003 y llegó a ser jefa de residentes entre 2007 y 2008; Carlos, que ingresó en el año 2007 y que al momento de la narrativa estaba realizando las prácticas externas de tercer año, y Cintia, que ingresó en 2008, que al momento de hacer la narrativa estaba finalizando su segundo año y que tuvo una posición de liderazgo en los últimos procesos del grupo.

En todo momento hemos utilizado guías y protocolos completamente abiertos tanto para el desarrollo de las narrativas como para el trabajo de coordinación psicológica del espacio de reflexión del grupo de residentes. La experiencia personal y los datos plasmados en el cuaderno de notas de las interacciones grupales fueron utilizados para la realización de las narrativas de los participantes así como en la narrativa del investigador. Tanto la jefa del grupo de residentes como los residentes que participaron en la investigación han sido informados con total puntualidad de los avances del trabajo, sus conclusiones y publicaciones.

\section{Marcos InSTITUCIONALES DEL GRUPO DE RESIDENTES}

Conviene comenzar con una breve exposición del marco institucional en el que toma forma el grupo de médicos residentes con el que hemos trabajado en la indagación. Se trata de un hospital general ubicado en una provincia argentina, inaugurado en los años 50 y que depende desde los 90 del Ministerio de Salud de la provincia, pero que estuvo previamente bajo la órbita de la Universidad Nacional. El hospital cuenta con los servicios de Clínica Médica, Cirugía General, Ortopedia y Traumatología, Tocoginecología, Pediatría, Unidad de Terapias Intensivas, Neonatología, Hospital de Día, Guardia y Sector Penitenciario.

El Servicio de Clínica Médica, además de prestar asistencia, es el marco de un posgrado. Este posgrado incluye, entre otras actividades, rotaciones (prácticas médicas) en consultorios externos y en los diversos servicios del hospital. Sus actividades de formación práctica incluyen pases de sala diarios con la presencia de los instructores de la carrera, o

\footnotetext{
${ }^{2}$ Para preservar el anonimato de los participantes en la investigación hemos modificado sus nombres.
} 
bien con la presencia de médicos especialistas, instructores y jefe del servicio, mientras que la formación teórica se apoya en una amplia serie seminarios diarios entre los que se incluye semanalmente el Grupo Balint de reflexión. El servicio cuenta con un jefe del servicio, un instructor y un tutor de residentes. Además, dado que es asiento de la Cátedra de Clínica Médica, cuenta con un profesor titular y dos profesores adjuntos. Este grupo de personas configura el staff responsable del servicio y de la formación del posgrado. El servicio cuenta además con un grupo de 13 médicos residentes, incluyendo al jefe de residentes. Este grupo de personas está involucrado en un proceso de aprendizaje-enseñanza, a la vez que sostiene la mayor parte de la presencia médica del servicio de cara a los usuarios.

Los residentes con los que hemos trabajado, ya sea en el Grupo Balint de reflexión sobre la práctica médica o en las narrativas, pertenecen al grupo de residentes. El trabajo se desarrolló entre los años 2003 y 2009. De este grupo cabe señalar tres cuestiones características: una, la importante influencia que sobre él ha tenido la crisis del ámbito de la salud, acrecentada por la problemática social y política que precede y continúa a la crisis del año 2001 en Argentina; dos, su continuado esfuerzo por posicionarse y enfrentar problemáticas que desbordan la estructura organizativa del hospital, $y$, tres, su empeño en defender el espacio de reflexión del Grupo Balint como lugar para la elaboración de una consciencia y acción grupal. Estas tres características son las que van a dar forma a las éticas que articulan los residentes a través de un proceso grupal cambiante y que son especialmente elaboradas en el ámbito del grupo de reflexión. Pasemos a ellas.

\section{HORIZONTALIDAD Y VERTICALIDAD: ÉTICAS DE AUTOGOBIERNO}

A pesar de la ausencia de un abordaje del papel de la ética en la literatura disponible sobre la participación no reglada de los médicos, la ética constituye, en la experiencia estudiada, un mecanismo de economía simbólica clave al posibilitar iniciativas y al facilitar la construcción de un proyecto que no se agota en lo inmediato. Comprendemos este enfoque como eticopolítico, en el sentido de que la ética conecta con la política a través de una serie de concepciones e intuiciones organizativas y políticas, manteniendo una estrecha relación con las políticas públicas acerca de la salud. A partir de aquí 
explicitaremos dos eticopolíticas opuestas creadas por este grupo de residentes como marco de su acción.

Estas éticas que emergen desde el espacio de reflexión del Balint combinan dos posiciones respecto del rol profesional, constituyendo y relacionando aspectos técnicos y político-institucionales de manera diversa. Esquemáticamente, podemos decir que una ética da rienda suelta a la incorporación dentro del rol médico de una serie de aspectos político-institucionales que emergen desde las situaciones enfrentadas cotidianamente por los médicos. Esta ética expresa, si tomamos como referente la "horizontalidad", una potencia de, y confianza en, procesos de autonomía (Castoriadis, 2010). La otra ética, por el contrario, tiende a segregar un dominio técnico al que limita el rol médico y a mantenerlo idealmente fuera de toda contaminación política. La "verticalidad" rechaza diversos aspectos políticos que, no obstante, parecen imponerse con fuerza. Los aspectos políticos en juego abarcan desde la propuesta de influir directamente sobre las decisiones que hacen al gobierno del hospital, pasando por las propias relaciones de poder internas al servicio de clínica, hasta la perspectiva de reclamo y medidas de fuerza de cara al Ministerio de Salud, apuntando así al sistema hospitalario en conjunto. En suma, constituyen acciones que contrarrestan la exclusión de los trabajadores de la salud en el seno del mismo sistema (Crojethovic, 2014). Comenzamos con esta segunda ética, la vertical, para tornarnos a continuación hacia la horizontalidad.

\subsection{Verticalidad}

La ética de la verticalidad refleja la estructura organizativa formal del hospital y se centra en una organización jerárquica que se fundamenta en tres argumentos: el conocimiento, el control y la representación.

El primero sustenta la organización vertical del hospital en una concepción jerárquica del conocimiento como representación. La naturaleza misma de la enfermedad establece los grados de adecuación y distancia de los conocimientos que la representan. De acuerdo a esto emergen los niveles de experticia a los que corresponden grados de decisión y poder, del que resulta una organización jerarquizada. Este argumento solo funciona efectivamente en el nivel del gobierno del hospital relativo a las unidades de trabajo, tales como los diversos servicios. El segundo argumenta que los agentes de la salud que trabajan en contacto directo con la comunidad no tienen control sobre 
el efecto de conjunto generado por sus prácticas y la organización vertical se sostiene llenando ese vacío mediante la acción de los estamentos jerárquicos. Este argumento, como el siguiente, justifica la función de la dirección y administración del hospital, a las que explícitamente no se considera con un saber sobre el trabajo médico concreto del hospital, entre otras razones debido a que son puestos políticos más que expertos. El último, que complementa al control, sostiene la verticalidad desde la distribución desigual de la autoridad del todo social obtenida por el Estado a través de la representación política. Al ordenar esta imagen jerárquicamente, sitúa la autoridad y la decisión política de toda la sociedad en los sectores de dirección del hospital. Es decir, la jerarquía del hospital se sostiene en la autoridad del todo social delegada por el Estado.

Siguiendo estos tres argumentos, la verticalidad propone separar el ejercicio profesional del ejercicio de la ciudadanía. La dimensión política de las prácticas de los agentes de salud se considera reabsorbida de antemano por el sistema de representación política y ejercida desde arriba por las direcciones del hospital. Dicho de otra forma, las decisiones generales que incumben a la función total y social del hospital no conciernen directamente a la relación entre los agentes de salud y la comunidad, sino solamente a las direcciones de hospital. Esta situación de purga de los aspectos políticos del rol médico se plantea a su vez como la mejor forma para desarrollar una cura que se basa en una enfermedad que se concibe como un objeto natural, independiente y pasible de representación objetiva. Esto es también reflejo de la idea de separación de la práctica política y la práctica de conocimiento como garantía de objetividad y de estabilización mutua (Callon, Lascoumes y Barthe, 2009). Sin embargo, la separación entre el conocimiento y lo político que sostiene la posición verticalista, como ya veremos con la emergencia de la ética horizontal, no sale indemne de los momentos de crisis socio-política. Dicho de otra forma, la crisis en la representación política, como la que emergió en Argentina en 2001, afecta a los modos de conocer y a la representación de los objetos, generando brechas donde germinan nuevas maneras de concebir el conocimiento y la práctica, que a su vez muestran con más claridad sus relaciones con planteamientos políticos. En este sentido, toda crisis social parece tener su faz epistemológica. 


\subsection{Horizontalidad}

La ética de la horizontalidad, que emergió desde un conjunto de prácticas no regladas que colaboró con sostener efectivamente el funcionamiento del hospital (Crojethovic, 2014), propone una organización no jerárquica basada en puntos de vista radicalmente diferentes. La horizontalidad sostiene una concepción no cosificada y no individualizada del conocimiento que imposibilita las estabilizaciones a partir de las cuales se establece la organización jerárquica de poder. Así plantea el conocimiento en circulación, con imposibilidad de localización en un elemento, y maneja una concepción grupal de este. Es esta concepción del conocimiento la que conduce a la búsqueda de una división "lógica" del trabajo, que en los hechos significa un replanteamiento de los roles formales en base a la práctica concreta.

Este planteamiento generaliza una serie de críticas a la estructura vertical que demuestran que en la práctica concreta no existe un funcionamiento eficaz basado en las diferencias de conocimiento. En particular sostienen que la mayor implicación y compromiso de los residentes con los casos concretos aplana las posibles diferencias de conocimiento con el staff estable de médicos responsables de la formación y del servicio. Y que cuando el staff estable utiliza el conocimiento desde una posición jerárquica obstaculiza la socialización de los lugares de saber y poder que en la práctica cotidiana requieren los residentes. Argumentan asimismo que en la práctica las diferencias entre los residentes y el staff emergen respecto a la mayor experiencia de estos últimos. Sin embargo, al concebir el marco en el que la experiencia es eficaz como un espacio de indeterminación, de ausencia de conocimiento, donde hay que vérselas con la arbitrariedad de la propia decisión en situaciones complicadas, la mayor experiencia no puede utilizarse para justificar una organización jerárquica respecto al conocimiento. Antes bien, sostienen que la mejor manera de enfrentar estas situaciones de elevada incertidumbre se basa en un debate grupal igualitario.

A su vez, el planteamiento horizontal sostiene que los que deben y pueden controlar los efectos de conjunto de las prácticas del hospital son los propios agentes de salud, a través de organismos de debate y decisión igualitarios. Esto se ve posibilitado porque los agentes de salud se han apropiado progresivamente de consideraciones económicas y administrativas en la base de su trabajo, entendiendo prácticamente lo 
que es una gestión hospitalaria. Asimismo, perciben que las direcciones y los estamentos jerárquicos parecen regirse por criterios que no se corresponden con los que emergen de la práctica y que, en los hechos, los mismos agentes de salud dirigen los efectos de conjunto de sus prácticas, aunque sea de manera lateral y espontánea, sin articular una reflexión acerca de ello.

Esta ética reincorpora no solo la perspectiva del gobierno del hospital como parte fundamental del rol profesional, sino una dimensión más directamente política implicada en una relación activa respecto al Estado. Esto parte de una crítica a las políticas estatales del momento dado que, al no contribuir a sostener y desarrollar la salud pública, no representaban a su creciente comunidad usuaria ni a los agentes de salud que la sostenían efectivamente con su trabajo. Lo que se produce es, entonces, un cuestionamiento de la imagen del Estado y permite arrogarse la autoridad social al representar las necesidades sociales.

En definitiva, la experiencia de los médicos residentes, constantemente empujados a resolver los problemas que enfrentan en todas sus dimensiones - problemas que no hacían más que evidenciar la "crisis de la división del trabajo" a nivel del hospital y del Estado como autoridad social-, muestra que lo que se encuentra en el centro de la propuesta horizontal es una articulación indisoluble de los aspectos políticos y técnicos.

\section{IMPLICACIONES DE LA EXPERIENCIA DE LOS RESIDENTES}

La falta de financiación estatal jugó un papel importante en el fracaso de las reformas de salud de los 90 , pero a esta falta se suman los agudos problemas de gobernanza, la escasa transparencia de la administración de los recursos y los grupos tradicionales de poder dentro de los modelos médico-hegemónicos. En este contexto emergieron nuevas e incipientes formas de gobernanza del hospital - formas abortadas con el paso del tiempo, como mostraremos en la siguiente sección-que mostraban una alianza con otros actores sociales y destacaban otro tipo de vínculos. Dado el desarrollo de estas prácticas, no podemos caracterizar ese momento como de ausencia de una bioética capaz de enfrentar los problemas cotidianos y de un estado de "atonía ética". De hecho, es posible detectar la emergencia de formas éticas alternativas provenientes de circuitos diferentes a los organismos médicos 
hegemónicos, algunas de las cuales, irrumpiendo desde abajo de la estructura hospitalaria, se afianzaban reapropiándose la práctica médica y la relación con los usuarios, así como tejiendo lazos con los organismos de democracia directa de la ciudadanía que emergieron en torno a la crisis de 2001.

En este sentido, los residentes articularon dos conjuntos de ideas y percepciones en torno a la horizontalidad y a la verticalidad que señalaban y disputaban respecto a la práctica de lo correcto desarrollando un plano ético. Este plano eticopolítico, y especialmente la horizontalidad, fue fruto de una acrecentada reflexividad y responsabilidad profesional. Sin embargo, no reflejaba la conexión de los individuos con los imperativos del gobierno bajo el principio liberal. Más bien reflejó la desconexión del poder estatal y la conexión con otros regímenes de poder alternativos surgidos de la pauperización y la precarización social propia de la crisis argentina de 2001. Asimismo, tampoco se trató simplemente de una experiencia ubicada en el marco estable de la oposición entre estructura formal e informal de una organización: los intersticios son por momentos demasiado grandes y la estructura demasiado floja.

Mostrando sin duda formas de participación no reglada que buscan ir más allá de la precarización institucional para plasmarse en renovada estructura, sobre el fondo de la impotencia y el cinismo que fue parte de la crisis del hospital, la eticopolítica horizontal implicó el desarrollo de una chispa esperanzada de potencia creadora que toma la forma de una acrecentada eticidad. Se trató de un evento no comprensible sin el afluir de la dinámica externa dentro del hospital, donde el desborde del hospital no significó solamente un exceso de trabajo respecto a la capacidad productiva, sino también el desarrollo de nuevas relaciones y discursos, temporalidades y afectaciones, que pasaron al menos momentáneamente a incorporarse al funcionamiento hospitalario. Este desborde tuvo efectos también a nivel epistemológico. Dentro del planteamiento horizontal encontramos una apertura hacia formas de conocimiento que toman en cuenta el efecto de las relaciones sobre el objeto de conocimiento, así como la indeterminación y la arbitrariedad del proceso. Este enfoque buscó recentrar la producción de conocimiento en la práctica misma y evitar la imposición de formatos previos sobre la enfermedad, apoyándose en un régimen de igualdad en la producción de conocimiento, cuestión que se vio plasmada 
especialmente respecto al diagnóstico y tratamiento en los pases de sala.

\section{TRES MOMENTOS}

Podemos distinguir tres momentos del grupo de médicos residentes estudiado entre 2003 y 2009, caracterizados por diferentes relaciones con el hospital y las situaciones que enfrentan en él y especialmente por un balance diferente de la operación y el desarrollo de las éticas profesionales de la horizontalidad y la verticalidad. La dinámica de estos tres momentos grupales, y en particular la plasmación de las dos eticopolíticas, remite a la conexión de los residentes con una serie de prácticas sociales que jugaron un papel en la construcción de la coyuntura política general del país en torno a la crisis del año 2001. Ello es aun más evidente en cuanto los procesos de horizontalidad y autonomía de los residentes no llegaron a plasmarse en una institución tal que configurara sus nuevas condiciones de creación, sino que al ir cambiando el contexto social y la subjetividad de los participantes en un sentido contrario a la horizontalidad, esta retrocede sin que nada se oponga a ello.

El fenómeno social conocido como "la experiencia de 2001" en Argentina o "el 2001" es extremadamente complejo. Uno de los rasgos de este momento puede ser caracterizado como un tipo de revuelta popular con acciones multitudinarias y elementos de democracia directa, en el marco de una pronunciada crisis estatal, que se prolongaron en una profunda movilización social que dio forma a un nuevo escenario político. En esta línea, algunos autores sitúan el 2001 entre una acción colectiva disruptiva y discontinua, llevada adelante en espacios públicos por multitudes y otros actores sociales y políticos, para expresar malestar o descontento por las condiciones sociales y políticas (López Maya, 2000; Tilly, 2002), y un movimiento social que rompió tendencialmente con los límites de compatibilidad del sistema y obligó a una reorganización del poder portando un fuerte carácter disruptivo e interpelador (Melucci, 1991). En una perspectiva más amplia se puede pensar este momento como parte de un "ciclo de acción colectiva antineoliberal" (Svampa, 2009) cuyo punto de eclosión se ubicaría en 1994 con la irrupción del zapatismo en Chiapas (México) y que comprendería, además, la Guerra del Agua en Cochabamba (Bolivia) durante el año 2000, los momentos de inflexión en Ecuador en el año 
2005 y nuevamente Bolivia en 2003 y 2006 (Svampa, 2009), en los que, como en el caso de Argentina, se dieron procesos de destitución de presidentes constitucionales, democráticamente electos, "derrocamientos por presión popular" (Darling, 2013: 313) en los que no se cancelaba la democracia, sino que se la radicalizaba. En el caso argentino, el año 2001 formaría parte de un ciclo de movilización social (Tarrow, 1998) que parece comprender momentos de pronunciada crisis de la democracia representativa, así como un auge de procesos de autodeterminación que rompieron en diversos grados con las estructuras organizativas clásicas (especialmente sindicatos y partidos políticos). Estos procesos generaron, además, vínculos que propiciaron actividades asamblearias, produjeron dispositivos de democracia directa y posibilitaron la emergencia de nuevos actores como los residentes organizados en asamblea a nivel provincial. Este proceso configuró en su complejidad las prácticas y conexiones que condicionaron a los residentes, en tanto estaban implicados de manera activa en los vínculos creados en esta etapa. Asimismo, el término horizontalidad, que acompañaba el estilo de relación y las acciones de esos momentos, es tomado por los residentes y recreado dentro del hospital.

Hacia el año 2006 parecía producirse una clausura de esta coyuntura. Por un lado, se generalizó una sensación de imposibilidad en torno a la construcción de una alternativa viable, operativa a escala nacional, a la impugnación de la democracia representativa y de la clase política en los espacios asamblearios y de democracia popular. Por otro lado, se puede hablar de un proceso paralelo en el que, a través de la implementación de una serie de cambios, los aparatos de Estado parecian obtener nuevamente una legitimidad que habian perdido. Todo ello en un contexto de transformación del escenario económico sensible para la población. Así, puede hablarse del comienzo de un ciclo de desmovilización (Tarrow, 1998) que porta también como sentido lo que aparece como las limitaciones de la apuesta horizontalista.

La "desconexión" de los circuitos de horizontalidad a partir de este momento de desmovilización y la recomposición de la figura del Estado parecen influir en la arquitectura ética de los diferentes momentos del grupo de residentes. La ética horizontal de los residentes ligada a la experiencia de 2001 parece retroceder en la misma medida que esa experiencia, y en ese proceso se pueden distinguir tres momentos. 
El primero, entre los años 2003 y 2005, coincidiendo aún con el ciclo de movilización de 2001, está marcado por un desborde de la implicación de los residentes con respecto a la organización formal del servicio y el hospital. Es el momento de mayor plasmación y desarrollo de la ética horizontal, durante el cual los residentes enfrentan el trabajo más técnico e inmediato con los pacientes, diseñando acciones que tienden a exceder los marcos de la estructura del hospital y durante el que aparece la estructura jerárquica del hospital como limitante. Los residentes buscan introducir modificaciones duraderas en la cotidianidad hospitalaria, a partir de, por un lado, la inquietud de dar continuidad a las modalidades de funcionamiento horizontal con el fin de instituirlas como marco organizativo estable $y$, por el otro, del desarrollo de una apuesta por ocupar por sí mismos, en lugar de obedecerlo, el lugar del poder político dentro del hospital que delega la responsabilidad de la asistencia únicamente en sus manos y naturaliza las malas condiciones de funcionamiento. "Se puede pensar un autogobierno horizontal asambleario del hospital, del conjunto, [...] sería excelente llegar a formar un gobierno asambleario" (narrativa de Mariana, jefa de residentes, 2009).

El segundo momento, entre los años 2006 y 2008, coincidiendo con el inicio del ciclo de desmovilización, aparece marcado principalmente por el desborde de la organización hospitalaria frente a la presión asistencial y solo en un segundo lugar por la acción de los residentes que desbordan con sus formas horizontales las estructuras jerárquicas, las cuales pasan gradualmente a ser significadas como la única posibilidad de organización del hospital: "nosotros seguimos el día a día como viene y vamos resolviendo los problemas hasta que alguno de esos problemas sea tan grave que nos tengamos que posicionar en alguna postura" (narrativa de Cintia, residente de segundo año, 2009).

Durante este momento se mantienen procesos de horizontalidad y autonomía desarrollados en el anterior: en las cuestiones organizativas del grupo y dentro el trabajo más técnico, en las decisiones sobre diagnósticos y tratamientos que aparecen fuera del marco del conocimiento, así como respecto a las acciones que conciernen a formas de reclamo conjunto que incluyen a todos los residentes del hospital o incluso de la ciudad, frente a instancias políticas. Sin embargo, los espacios que hacen a la reflexión grupal, como el Grupo Balint, pierden interés y fuerza para los residentes, así como la importancia 
previamente manifestada del "decidir y llevar a la práctica lo decidido". Los residentes ya no creen en un gobierno horizontal del hospital: aun concibiéndolo como posibilidad, no se desea:

\begin{abstract}
Sería una locura organizar todo el hospital de una manera más horizontal, de manera de que haya mayor igualdad entre los distintos agentes a la hora de las decisiones. Yo creo que lo jerárquico debe existir (narrativa de Carlos, residente de tercer año, 2009).
\end{abstract}

Vemos cómo la ética de la verticalidad va ganando terreno en la acción paulatinamente. Se produce así una desarticulación del proyecto construido en el momento anterior, donde la acción "no reglada" tiende a reproducir la precariedad del hospital:

Se va haciendo entonces lamentablemente sin una estructura de fondo y es difícil, porque se va armando el día a día en lo que va pasando, en lo que vas haciendo (...) lo más común es organizarse con lo que hay, en condiciones de gran fragilidad (narrativa de Carlos, residente de tercer año, 2009).

No hay una continuidad de las cosas y a una le llega a cansar (narrativa de Cintia, residente de segundo año, 2009).

El tercer momento, que comprende el año 2009, está marcado por el desborde del grupo de residentes debido en parte a la presión asistencial y en parte a las propias dinámicas internas del grupo. Ambos factores configuran una situación imposible de frenar para retomar desde ahí una posición activa:

No llegamos casi a decidir y actuar en las cuestiones pequeñas y mucho menos podemos pasar de ellas a las más grandes (...) No estamos teniendo espacios específicos de debate, reflexión y decisión conjunta (...) Necesitamos un espacio para poner las cosas en limpio (...) dado que estamos muy estresados por esas cosas (narrativa de Cintia, residente de segundo año, 2009).

El grupo está fragmentado, los residentes se encuentran enfrentados entre sí, incapaces de resolver lo que aparece como conflictos interpersonales, cargando individualmente con los problemas. Requieren de la operación de los instructores desde un lugar jerárquico, aunque esto por momentos genere resistencias, para crear un marco que permita tomar y sostener decisiones sobre lo organizativo interno del grupo, así como sobre diagnósticos y tratamientos (en particular lo que aparecía bajo la ética horizontal en el momento anterior). Incluso es 
requerida una acción verticalista del jefe de residentes para rearmar el grupo y volver a coordinar su acción y recuperar el posicionamiento grupal en los reclamos frente al Ministerio de Salud. La ética verticalista parece predominar en el grupo y a la vez empieza a concebirse la residencia como algo que hay que pasar de manera rápida, escéptica y aséptica evitando ser dañado por las circunstancias. La ética de la horizontalidad está prácticamente ausente, ya no aparece como un pilar del grupo. La falta de participación y de cooperación en diversos aspectos de la residencia se relaciona con el descreimiento en la influencia que los residentes pueden tener sobre las jerarquías del hospital (lo cual en el primer momento generaba una participación horizontal). En este punto los residentes ya no conciben como posible un gobierno horizontal de los agentes de salud sobre el hospital y por momentos parecen quedar en una posición de dependencia directa respecto a la jerarquía hospitalaria:

En este momento hay poco y nada de decisión y determinación desde los propios residentes (...). El sello final lo pone (...) uno de los instructores del servicio (...), todo termina pasando por él (...). Se hace lo que él dice (Narrativa de Cintia, residente de segundo año, 2009).

\section{PRÁCTICAS DE LA HORIZONTALIDAD, BALINT Y CONFRONTACIÓN}

El grupo de residentes logró el mayor grado de desarrollo y plasmación de la ética de la horizontalidad entre los años 2003 y 2006. Ese momento implicó ciertas prácticas de organización, decisión y poder que giraban en torno al espacio de reflexión sobre la práctica médica del grupo de residentes, el Grupo Balint, y ciertos ejercicios de confrontación de los residentes con los instructores, jefes del servicio, la dirección del hospital y el Estado.

El espacio Balint para la reflexión de la práctica del servicio adquirió, de hecho, un lugar privilegiado: llegó a ser el espacio de debate y decisión del grupo de residentes que suplantaba los espacios de decisión externos que operaban sobre ellos y que permitía articular grupalmente sus prácticas no regladas. Las relaciones que se daban en él estaban definidas explícitamente por la horizontalidad, en referencia a una igualdad en cuanto al derecho a la palabra, a la decisión sobre las cuestiones del grupo y a la hora de llevar adelante esas decisiones en la práctica. Se definía así un tipo de relaciones de poder igualitarias entre 
los residentes, que involucraba un grado de flexibilidad frente a las situaciones, indeterminación y libertad que promovía la implicación en el debate. La centralidad de este espacio frente a otros del servicio se debía a que era el que más libertad proporcionaba a los residentes y les permitía instaurar una distancia reflexiva a partir de la cual generar una posición crítica sobre sí mismos para posicionarse en conjunto. En eso se diferenciaba de la práctica médica más técnica en la que estaban sujetos al orden del conocimiento, de la técnica, de la estructura vertical del hospital, impuesta bajo la presión de la propia labor y que no permitía un espacio de reflexión sobre los aspectos de conjunto del servicio. Si bien llegaban a una discusión horizontal de los casos en los pases de sala, postulando igualdad en el conocimiento, se encontraban sujetos, no obstante, a algunos ejercicios de autoridad por parte de los instructores. Lo significativo del espacio Balint es que allí los residentes tomaron decisiones que se llevaron a la práctica fuera de él, expandiendo su régimen de relaciones y generando representaciones que transformaban tanto el Balint y los pases de sala como la práctica más técnica en general. Estas representaciones contribuyeron a difundir la ética de la horizontalidad en torno a la idea de que el mejor régimen para la práctica era la igualdad de todos los agentes de salud implicados en la labor común.

Las decisiones tomadas en el espacio Balint abarcaban el conjunto de las cuestiones organizativas del trabajo de los residentes en el servicio, es decir, desde cuestiones internas como las guardias de los residentes, las fechas de los seminarios, los pases de sala, hasta cuestiones externas como las relaciones con otros servicios, la realización o no de prácticas en ellos, o haciéndolas de una manera específica. También abarcaban las relaciones con la dirección del hospital, a partir de decisiones y acciones que tenían por fin "imponerles" tratamientos específicos y mejores condiciones para los pacientes, conseguir mejores condiciones laborales en términos de insumos, espacio y recursos o buscar influir en la distribución del presupuesto desde consideraciones económicas y administrativas del propio grupo. Asimismo, en este espacio se tomaban decisiones que afectaban las relaciones a nivel del Estado mediante acciones de lucha y de reclamo con el conjunto de los residentes del hospital y la ciudad dirigidos al Ministerio de Salud, bajo el lema de la defensa de la salud pública, de la defensa de sus condiciones salariales y del reclamo de insumos. El peso de estas decisiones hizo que el Balint se 
convirtiera en el espacio de decisión y diseño de los otros espacios de los residentes y condujo a que la organización jerárquica empezara a reducirse: la práctica médica más técnica dejaba entonces de ser algo vertical y se convertía en una división de tareas, según los años de residencia,y mantenía igual poder de decisión para todos a la vez que se proyectaba horizontalidad en los pases de sala, sobre las decisiones del diagnóstico y tratamientos, lo que generaba una mayor implicación y un debate igualitario en el que el lugar jerárquico de los instructores se dejaba de lado. Todo ello se acompañaba del desarrollo de una posición política dentro del hospital que criticaba la transferencia de trabajo hacia los residentes por parte de algunos médicos de planta, la carencia de cobertura social y la situación de los residentes ad honorem (explicitando a su vez la condición legal de los residentes), así como las extensas jornadas laborales. Además, llevaban adelante una fuerte crítica a la distribución y al uso del presupuesto dentro del hospital, así como a la consecuente falta de insumos básicos, espacio y recursos de formación, y, en general, la falta de presupuesto para la salud pública.

Otro elemento destacado en este momento de horizontalidad fueron los ejercicios de confrontación de los residentes que afectaban diversos asuntos y agentes. Esta confrontación se fundamentaba mayormente en que diversos agentes (los instructores, los jefes del servicio, la dirección del hospital y el Estado) imposibilitaban a los residentes hacerse cargo de sus situaciones y autodeterminarse según la práctica que consideraban correcta, lo que obstaculizaba la horizontalidad. Así, específicamente, los residentes se enfrentaban al rol formal de los instructores y del profesor titular del servicio determinando las cuestiones organizativas de guardias, seminarios, pases de sala y rotaciones, y llegaban también a excluir a un instructor del espacio del Balint y a sostener diagnósticos y tratamientos en conflicto con ellos. A su vez, se excluía del Balint al jefe del servicio y al profesor titular debido a que obstaculizaban el debate entre los residentes. Además, se los pasaba por alto al dirigirse directamente a los jefes de otros servicios y a la dirección del hospital desde las decisiones horizontales del grupo de residentes. Específicamente, se confrontó al jefe del servicio diciéndole que no se lo consideraba tal debido a que no tenía papel alguno en la práctica médica ni en los aspectos políticos del servicio y por intentar imponer un jefe de residentes que no había sido elegido por el grupo de residentes (elección que formalmente correspondía a instructores y 
jefes). Asimismo, se confrontó a los jefes de otros servicios del hospital para detener la derivación indiscriminada de pacientes hacia el servicio de clínica, negociando a partir de las rotaciones (trabajo en otros servicios) y buscando el diálogo con los residentes de esos servicios en torno a problemas concretos. Con respecto a la dirección del hospital, se rompía la incomunicación al dirigirse directamente hacia ella, saltando el rol del jefe del servicio y llevando alli una serie de problemáticas que este parecía no querer ver, lo que llevó a que se lograra la autorización de tratamientos y mejores condiciones para los pacientes, así como insumos, espacios y recursos. También se la confrontaba participando en las asambleas del hospital y representando al hospital en la asamblea interhospitalaria de la ciudad, así como en las medidas de fuerza llevadas adelante. La confrontación era también contra el Estado al imponer reclamos en la arena pública confluyendo con los médicos residentes del hospital o de toda la ciudad en movilizaciones, paros y medidas de lucha de gran creatividad, a partir de los cuales lograron algunas de sus demandas.

Estas prácticas de organización, decisión y poder de los residentes formaron parte de la fase de movilización de 2001 hacia dentro y hacia fuera del hospital. El objetivo de estas prácticas era una modificación duradera de la estructura del servicio y del hospital. La ética que emergió en solidaridad con estas prácticas se configuró como una eticopolítica "de abajo hacia arriba" en tanto se generaba desde las prácticas cotidianas del hospital, creciendo desde la autoorganización de los residentes en relación directa con la comunidad de usuarios y abarcando las relaciones dentro del hospital y con el Estado a través del Ministerio de Salud.

\section{CONCLUSIONES}

El caso que hemos abordado nos ha permitido conocer algunos aspectos no conocidos de la crisis de la salud argentina en torno al año 2001. En particular nos ha interesado analizar cómo, frente a la amplia precarización de los hospitales y en un contexto de una estructura organizativa desbordada, algunos sectores del personal sometidos a una fuerte presión y particularmente excluidos pueden llegar a organizarse y desarrollar prácticas por fuera de la estructura formal como una vía para hacer frente a la situación. Pero estas formas de organización y estas prácticas son también mecanismos que les permiten tener un efecto 
concreto sobre el gobierno del hospital, así como plantear otras formas de gobierno de este, especialmente mediante el reemplazo de las instancias jerárquicas por un gobierno asambleario. Vimos además la emergencia de formas éticas alternativas - la horizontalidad-, así como el papel central que pueden jugar operando como mecanismo económico clave en el sostén, la guía y la disputa del marco de prácticas no formales, haciéndolas coherentes en un planteamiento que busca ir más allá de la precariedad. Asimismo, señalamos la manera en que estas innovaciones organizativas y éticas pueden relacionarse con una serie de vínculos con sectores diferentes a los tradicionales del modelo médico-hegemónico, por ejemplo, la articulación de conexiones con asambleas ciudadanas y la organización asamblearia de los residentes de toda la ciudad. De esta manera, hemos destacado que el contexto de crisis político social, y en especial de crisis de representación política, ingresa al hospital a su manera, no solamente como negatividad sino también portando nuevas posibilidades abiertas por las respuestas innovadoras que socialmente se han dado frente a la crisis.

El concepto de eticopolítica ha demostrado ser fundamental para comprender la acción de los residentes, ya que abarca en particular el conjunto de ideas que plasmaron, la acrecentada reflexión en torno a la práctica, a las relaciones de poder y a la política y la elaboración de un marco de autonomía. En este sentido, el concepto es particularmente interesante para pensar la emergencia de nuevos actores y prácticas en contextos de precariedad de la salud y de crisis estatal y social. Esto se debe a que permite conectar la importante dimensión ética del campo de la salud y la autonomía de los agentes con la situación de apertura e incertidumbre propia de la crisis política, así como con la creatividad emergente de las acciones colectivas y de los movimientos sociales, concibiendo un espacio para el juego de estos factores. Además, el concepto posibilita focalizar momentos de gran desestructuración donde proliferan fragmentos de estructura y la fluidez de prácticas informales, así como procesos de pasaje hacia nuevos momentos de mayor estructuración. El ingreso en el hospital de nuevas relaciones y discursos, temporalidades y afectaciones ayuda a comprender la posibilidad de reposicionamiento activo de los sujetos en momentos de gran incertidumbre, precariedad y fragilización. Es también de señalar que la experiencia investigada nos ha llevado a separar el concepto de eticopolítica del gobierno liberal en tanto la eticopolítica observada no 
refleja el buen gobierno liberal, sino un momento de desmantelamiento, de crisis, donde aparece la conexión con otros actores sociales tradicionalmente excluidos.

En suma, el concepto de eticopolítica puede ser útil para investigar formas de gobernanza emergentes y nuevas éticas en contextos de extrema precariedad y fluidez institucional, atendiendo a la conexión política, a las nuevas alianzas, y dando cuenta de formas de gobernanza en libertad sin conexión inmediata con un modo de gobierno liberal, sino con una multiplicación de formas de poder que emergen desde la base.

\section{Bibliografía}

Balasch, M., y Montenegro, M. (2003). Una propuesta metodológica desde la epistemología de los conocimientos situados: Las producciones narrativas. Encuentros en Psicología Social, 1/3, 4448.

Belmartino, S. (2005). Una Década de Reforma de la Atención Médica en Argentina. Salud Colectiva, 1(2), 155-171.

Callon, M., Lascoumes, P., y Barthe, Y. (2009). Acting in an Uncertain World: An Essay on Technical Democracy. Cambridge: MIT Press.

Castoriadis, C. (2010). Autonomía y alienación. En La institución imaginaria de la sociedad (pp. 160-182). Buenos Aires: Tusquets.

Cetrángolo, O., y Devoto, F. (1998). Reformas en la política de salud en la Argentina durante los años noventa con especial referencia a la equidad. Argentina: CECE.

Crojethovic, M. (2014). Los efectos de la participación no regulada en los hospitales públicos de la provincia de Buenos Aires. Argentina. KAIROS, Revista de Temas Sociales, 33, 2-19.

Darling, V. (2013). Reflexiones sobre el poder destituyente de los movimientos sociales en América Latina. Andamios, 10(21), 303320.

Federación Europea de Medicina Interna; American College of Physicians; American Society of Internal Medicine (ACP-ASIM) y American Board of Internal Medicine (2010). Profesionalismo médico en el nuevo milenio: una declaración para el ejercicio de la medicina. Revista Argentina de Cardiología, 78(4), 364-366. 
Flyvbjerg, B. (2004). Cinco malentendidos acerca de la investigación mediante los estudios de caso. Revista Española de Investigaciones Sociológicas, 106/04, 33-62.

Foucault, M. (1988). El sujeto y el poder. Revista Mexicana de Sociología, 50(3), 3-20.

Grau, A., Flichtentrei, D., Suñer, R., Prats, M., y Braga, F. (2009). Influencia de factores personales, profesionales y transnacionales en el sindrome de burnout en personal sanitario hispanoamericano y español. Revista Española de Salud Pública, 83, 215-230.

Guba, E., y Lincoln, Y. (1994). Competing paradigms in qualitative research. En N. Denzinn y Y. Lincoln (eds.). Handbook of qualitative research (pp. 105-117). Thousand Oaks: Sage.

Haraway, D. (1995). Conocimientos situados: la cuestión científica en el feminismo y el privilegio de la perspectiva parcial. En Ciencia, Cyborgs y mujeres: la reinvención de la naturaleza (pp. 313-346). Madrid: Cátedra.

Katz, I. (2004). Argentina Hospital. El rostro oscuro de la salud. Buenos Aires: Edhasa.

Katz, J., y Muñoz, A. (1988). Organización del sector salud: puja distributiva yequidad. Buenos Aires: CEPAL.

Litewka, S. (2010). Calidad, sistema de salud y gobernanza. El caso argentino. Acta Bioethica, 16(2), 148-154.

López Maya, M. (2000). La protesta popular en la Venezuela contemporánea. Enfoque conceptual, metodológico y fuentes. En J. Rodríguez (Comp.). Visiones del oficio. Historiadores venezolanos en el siglo XXI (pp. 399-412). Caracas: Academia Nacional de la Historia/FHE-UCV.

Melucci, A. (1991). Qu'y a-t-il de nouveau dans les "nouveaux mouvements sociaux"?. En L. Maheu y A. Sales (Eds.), La recomposition du politique (pp. 129-162). Montreal: Presses de I'Université de Montréal.

Pujol, J., Montenegro, M., y Balasch, M. (2003). Los límites de la metáfora lingüística: implicaciones de una perspectiva corporeizada para la práctica investigadora e interventora. Política y Sociedad, 40(1), 5770.

Ribé Buitrón, J. M. (2012). ¿Qué fue de los tradicionales grupos Balint? En defensa de la perspectiva Balint y una metodología adaptada en 
los espacios de coordinación con Atención Primaria. Norte de Salud Mental, X(44), 13-27.

Rose, N. (2001). The politics of life itself. Theory, Culture y Society, 18(6), 130.

Svampa, M. (2009). Protesta, movimientos sociales y dimensiones de la acción colectiva en América Latina. En Jornadas de Homenaje a $C$. Tilly. Madrid: Universidad Complutense de Madrid-Fundación Carolina.

Tarrow, S. (1998). El poder en movimiento: los movimientos sociales, la acción colectiva y la política. Madrid: Alianza.

Tilly, C. (2002). Repertorios de acción contestataria en Gran Bretaña: 1758-1834. En M. Traugott (Ed.). Protesta social. Repertorios y ciclos de la acción colectiva (pp. 1-17). Barcelona: Hacer.

Van Wynsberghe, R., y Khan, S. (2007). Redefining case study. International Journal of Qualitative Methods, 6(2), 80-94.

Yin, R. K. (1994). Case study research. Design and methods. Thousand Oaks-Londres-Nueva Delhi: Sage. 